\title{
The lithium history of NGC 6397
}

\section{Francesca Primas, ${ }^{1}$ Karin Lind,${ }^{1}$ Corinne Charbonnel, ${ }^{2}$ Frank Grundahl ${ }^{3}$ and Martin Asplund ${ }^{4}$}

\author{
${ }^{1}$ European Southern Observatory, Karl-Schwarzschild-Str. 2, D-85748, Garching, Germany \\ email: [fprimas, klind] @eso.org \\ ${ }^{2}$ Geneva Observatory, 51 chemin des Mailettes, 1290 Sauverny, Switzerland \\ email: Corinne.Charbonnel@obs. unige.ch \\ ${ }^{3}$ Department of Physics \& Astronomy, Aarhus University, Ny Munkegade, \\ 8000 Århus C, Denmark \\ email: fgj@phys.au.dk \\ ${ }^{4}$ Max-Planck-institut für Astrophysik, Karl-Schwarzschild-Str. 1, D-85748 Garching, Germany \\ email: asplund@MPA-Garching.MPG.de
}

\begin{abstract}
The primordial lithium abundance inferred from WMAP and standard Big Bang nucleosysnthesis is approximately three times higher than the plateau value measured in old metal-poor Population II stars, suggesting that these stars have undergone atmospheric Li depletion. To constrain the physics responsible for such depletion, we conducted a homogeneous analysis of a large sample of stars in the metal-poor globular cluster NGC 6397, covering all evolutionary phases from below the main-sequence turnoff to high up the red-giant branch (RGB). The dwarf, turnoff, and early subgiant stars form a thin abundance plateau, with a sharpe edge in the middle of the subgiant branch, where Li dilution caused by the inward extension of the convective envelope starts (the beginning of the so-called first dredge up). A second steep abundance drop is seen at the RGB bump, again highlighting the need for the onset of nonstandard mixing in this evolutionary phase. Moreover, by also measuring the sodium abundances of the targets, we have gained insight into the degree of pollution by early cluster self-enrichement, and may separate highly polluted, Li-poor and Na-rich stars from stars formed from pristine material. Our observational findings strictly limit both the extent of lithium surface depletion, which in turn constrains the efficiency of mixing below the outer convection zone, and the resulting spread in lithium abundance in metal-poor turn-off stars.
\end{abstract}

Keywords. stars: abundances, stars: atmospheres, stars: evolution, globular clusters: individual (NGC 6397)

\section{Introduction}

From the time of its discovery in the stellar atmospheres of metal-poor Population II stars (Spite \& Spite 1982), lithium has been considered a key diagnostic to test and improve our knowledge of the primordial Universe, stellar interiors and evolution, and spallation physics (especially so if lithium abundances are combined with beryllium and boron). Lithium-7 is one of the four primordial isotopes which were formed in observable quantities by nuclear reactions during the first minutes after the Big Bang (e.g., Olive et al. 2000; and references therein). Together with deuterium, helium-3, and helium-4, knowledge of its primordial abundance provides an important observational constraint on the baryon-to-photon ratio $\eta \propto \Omega_{\mathrm{b}} h^{2}$, which is the main free parameter of standard Big Bang nucleosynthesis (SBBN).

Despite numerous observations of lithium in stars, we still lack a consistent picture and the primordial abundance remains under debate. Prior to the discovery of a notably 
flat and constant $\mathrm{Li}$ abundance- $A(\mathrm{Li})=12+\log (N(\mathrm{Li}) / N(\mathrm{H})) \approx 2.2$-among Galactic warm halo dwarf stars, spanning a wide range of effective temperatures and metallicities (the so-called Spite plateau), it was generally believed that the primordial Li abundance was in the range $A(\mathrm{Li})=3.0-3.3$, which is the value measured in meteorites and also the maximum value detected in Population I stars, both in the field and in open clusters (cf. Boesgaard \& Steigman 1985). If this were the case, the close-to-constant but lower value of lithium along the Spite plateau would have required that all of the oldest stars in our Galaxy had suffered uniform depletion by about a factor of 10. If, instead, the plateau value represented the amount of Li produced during the Big Bang, the Galaxy should have been enriched in its Li content by a factor of at least 10 since its birth.

During the last decade, observations of cosmic-microwave-background (CMB) anisotropies, most particularly through the Wilkinson Microwave Anisotropy Probe (WMAP) experiment (Bennet et al. 2003; Spergel et al. 2003), have allowed unprecedented precision as regards the determination of the baryon density $\Omega_{\mathrm{b}} h^{2}$ and seem to reveal that the truth about Li lies between the two extreme solutions discussed above. With the most recent value of $\Omega_{\mathrm{b}} h^{2}$ derived from the five-year release of WMAP data $\left(\Omega_{\mathrm{b}} h^{2}=\right.$ $0.02273 \pm 0.00062$; Dunkley et al. 2009), updated SBBN predictions provide a precise determination of the primordial abundances of the light elements $\mathrm{D},{ }^{3} \mathrm{He},{ }^{4} \mathrm{He}$, and ${ }^{7} \mathrm{Li}$ that can be compared with observations in low-metallicity environments. For ${ }^{7} \mathrm{Li}$, the CMB-derived primordial abundance is clearly higher $(A(\mathrm{Li})=2.72 \pm 0.06)$ than the current determinations in low-metallicity halo stars. This result seems to be very robust with respect to the nuclear uncertainties on the SBBN reactions. An alternative explanation is that the $\mathrm{Li}$ abundance seen on the surface of halo stars is not the pristine value, but that these stars have instead undergone surface-lithium depletion at some point during their evolution.

That lithium and other elements should be prone to atomic diffusion and thus be depleted from stellar atmospheres by gravitational settling was already predicted by Michaud et al. (1984). However, without the existence of macroscopic mixing acting below the outer convection zone, a thin and close-to-flat Spite plateau cannot be reproduced by stellar-structure models (Richard et al. 2005). As of today, the physical process behind the mixing has yet to be identified. So, the question is, can we - through detailed observations and analysis of lithium in Population II stars - place rigid constraints on the nature and efficiency of this nonstandard mixing?

\section{Our project: definition and goals}

To constrain the physics responsible for surface depletion, one needs to accurately establish the behaviour of surface $\mathrm{Li}$ abundance as a function of stellar effective temperature and evolutionary phase. Globular clusters are ideal environments, since relative stellar parameters can be determined precisely. NGC 6397 is one of the most extensively observed globular clusters and its lithium content has been documented down to the magnitude of the cluster turnoff point in several studies (Pasquini et al. 1996; Castilho et al. 2000; Thévenin et al. 2001; Bonifacio et al. 2002; Korn et al. 2007), although with poor number statistics at faint magnitudes.

Therefore, we decided to target this cluster by observing a large sample of stars and conduct a homogeneous analysis, fully covering all evolutionary phases from below the main-sequence turnoff (around $V=17 \mathrm{mag}$ ) to high up the RGB (around $V=11.5 \mathrm{mag}$ ). In total, 349 stars were observed, 160 of which sample the subgiant and the red-giant branch $(80$ each), and the rest $(\sim 190)$ sample the turnoff point. All our targets were selected from Strömgren uvby photometry. The sample was observed with FLAMES, the 
high-resolution multiplex facility at the VLT/UT2, using the UVES+GIRAFFE/ MEDUSA combined mode. For approximately one third of the sample we also covered the Na doublet at $8183 \AA$ and $8194 \AA$, which is a fundamental ingredient to properly disentangle between in situ depletion and pre-enrichment effects (by testing the existence of a $\mathrm{Li}-\mathrm{Na}$ anticorrelation, if any). More details about the observations and the reduction of the data set can be found in Lind et al. (2009b).

\section{Stellar parameters}

We derived effective temperatures from uvby photometry, gravities from the common relation between luminosity and mass (obtained from the cluster isochrone), and metallicity from measuring the equivalent widths of several FeI and FeII lines. The most critical parameter for lithium-abundance determination is the effective temperature: $\pm 100 \mathrm{~K}$ in effective temperature corresponds to approximately \pm 0.07 dex in lithium abundance.

The colours were translated to effective temperatures using the MARCS synthetic-colour calibration of Önehag et al. (2009), as well as the infrared-flux-method calibrations of Ramírez \& Meléndez (2005) and Alonso et al. (1996, 1999). In the turnoff region, our targets show a spread of $0.015 \mathrm{mag}(1 \sigma)$ in $v-y$ colour around the cluster sequence. With a sensitivity of $v-y$ of $37 \mathrm{~K}$ per $0.01 \mathrm{mag}$, this corresponds to a spread in effective temperature of $56 \mathrm{~K}$. The more temperature-sensitive $b-y$ colour has a typical spread of $0.01 \mathrm{mag}$, which corresponds to $95 \mathrm{~K}$. Moreover, the target colours could also be affected by differential reddening, and we circumvented the problem by constructing colour-magnitude fiducial sequences and interpolated the visual magnitude of each target onto the sequence. This is done to reduce artificial spreads in effective temperature caused by reddening and observational uncertainty, which would propagate into a significant uncertainty in lithium abundance.

We have compared our temperatures with the values determined in the recent study by González Hernández et al. (2009) for a group of subgiants and main-sequence stars in NGC 6397. These authors use profile fitting of the $\mathrm{H} \alpha$ wings to infer effective temperatures, with 3D hydrodynamical model atmospheres. The $\mathrm{H} \alpha$-based values have a systematic offset towards higher temperatures by approximately $80 \mathrm{~K}$, but the relative agreement is good, considering the fully independent methods adopted. Their temperatures show a scatter around the cluster sequence of $105 \mathrm{~K}$ for the subgiants and $85 \mathrm{~K}$ for the dwarfs, which may be a reasonable estimate of the true spread.

\section{Abundance analysis}

We carried out the abundance analysis using 1D, LTE (local thermodynamic equilibrium), plane-parallel and spherical MARCS model atmospheres (Gustafsson et al. 2008). Spectral synthesis was employed for the lighter elements (up to $\mathrm{Al}$ ), while equivalentwidth measurements were used for all other elements (e.g., Fe).

Lithium abundances were derived from minimisation of the $\chi^{2}$ distance between synthesis and observations for a total of 305 stars (including a few upper limits). Sodium abundances were derived using the same technique, but for a subset of 117 stars (again, including upper limits). Both $\mathrm{Li}$ and $\mathrm{Na}$ abundances were corrected for non-LTE effects. For Li, we calculated the non-LTE corrections according to the recent study by Lind et al. (2009a); for Na we applied non-LTE corrections following the work of Mashonkina et al. (2000). 
For completeness, it is important to mention that we derived many more abundances of $\alpha$, iron-peak and heavy elements. These will be presented in a forthcoming paper (Lind et al., in prep.) which will tackle the chemical evolution of this globular cluster.

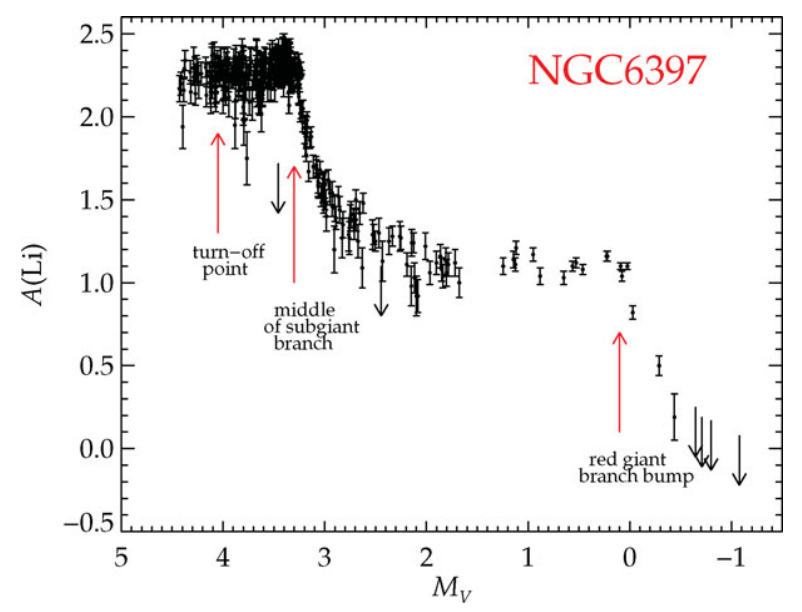

Figure 1. Non-LTE Li abundances versus absolute visual magnitude for all our NGC 6397 targets. Labels identify some of our main findings as described in the text.

\section{Results and implications}

The dwarf, turnoff, and early subgiant stars in our sample form a thin abundance plateau, with a sharp edge in the middle of the subgiant branch, where the Li dilution caused by the first dredge up sets in. A second steep abundance drop is seen at the RGB bump (cf. Figure 1), which likely is due to the onset of thermohaline mixing (Charbonnel \& Zahn 2007).

A considerably large spread in $\mathrm{Na}$ abundance $(\sim 1 \mathrm{dex})$ confirms the findings of Carretta et al. (2005), that NGC 6397 has suffered from intracluster pollution in its infancy. The most lithium-poor objects show extreme Na enhancement (cf. Figure 2), and we deduce that these objects have suffered heavy pollution from a previous generation of stars. This is a very important result on its own, since it rules out other explanations for the $\mathrm{Li}$ deficiency (such as, e.g., abnormal rotational velocity or binarity; see, e.g., Ryan et al. 2001). Second, detecting lithium in highly polluted, sodium-rich stars points to the need for mixing with pristine matter, since stellar ejecta presumably are Li-free (e.g., Prantzos \& Charbonnel 2006).

The Li-Na anticorrelation (cf. Figure 2) relies on a handful of the most polluted objects to be significant, i.e., it appears that $\mathrm{Li}$ abundances are essentially unaffected by relatively large degrees of pollution. No significant difference in the behaviour of mean lithium abundance with evolutionary phase is found if polluted stars (assumed to be those with a $A(\mathrm{Na}) \geqslant 3.9)$ are separated from nonpolluted objects. Moreover, the spread in abundance among nonpolluted objects with similar stellar parameters $(\sigma=0.09 \mathrm{dex})$ is consistent with observational errors, and does not allow for significant star-to-star differences.

Figure 3 illustrates how the mean lithium abundance varies with visual magnitude in the cluster and also compares our findings to other, similar studies of NGC 6397. A feature seen in all three of the most recent studies is that subgiants appear more lithium-rich than less evolved stars, as already suggested by Charbonnel \& Primas (2005) for field stars. Our observations reveal a small upturn in Li abundance, located just prior to the 


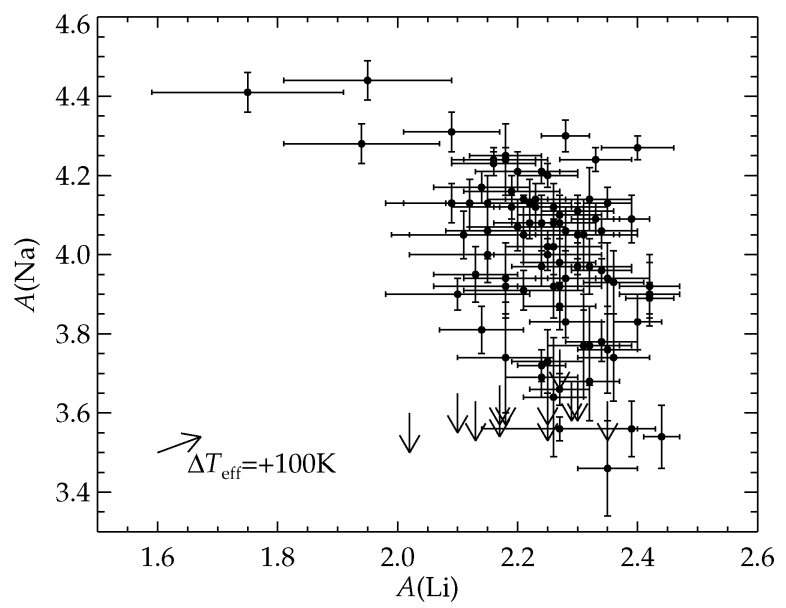

Figure 2. Non-LTE Li and Na abundances for the subsample of 100 dwarfs and subgiants with $M_{V}>3.3 \mathrm{mag}$. The arrows mark Na-abundance upper limits. If the effective temperature of a target is overestimated by $100 \mathrm{~K}$, its corresponding location in this diagram will shift in the direction of the tilted arrow (or vice versa).

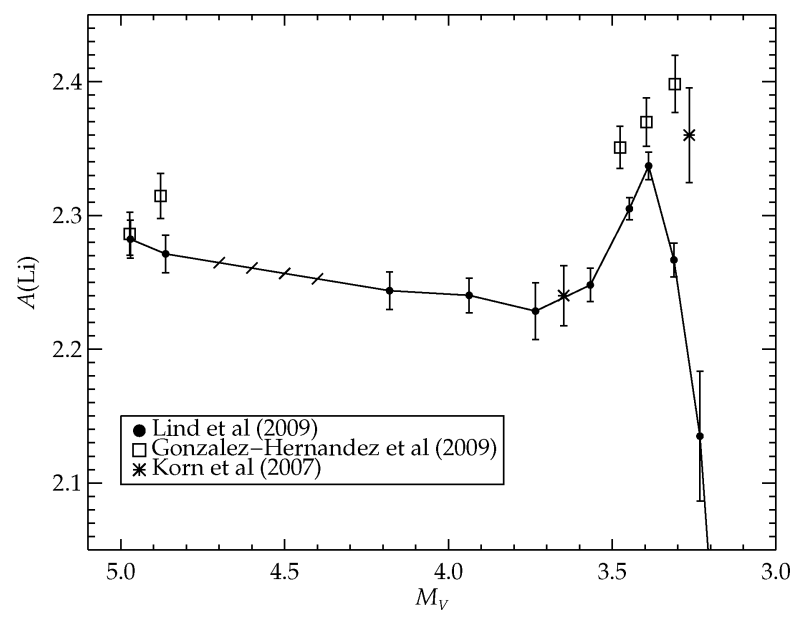

Figure 3. Group-averaged lithium abundance versus absolute visual magnitude for our targets and two other studies of NGC 6397, as indicated by the different symbols. The higher lithium abundances inferred by González Hernández et al. can be traced to their warmer effective-temperature scale $(80 \mathrm{~K})$.

stage where Li is strongly diluted, which may indicate that an overabundance is formed above Li-free layers in the stellar interior.

Since the effective-temperature determination has large influence on the derived lithium abundances, and as of yet no true consensus has been reached regarding the reliability of different photometric and spectroscopic methods for determining this fundamental stellar parameter for metal-poor turnoff stars (especially not within $100 \mathrm{~K}$ accuracy), trends of lithium with effective temperature are likely to contain a certain bias. Whereas Figure 3 points to a general agreement between different studies, a similar plot with effective temperature as reference axis would show significant discrepancies, as was also pointed 
out by González Hernández et al. (2009). Stellar luminosity has, of course, no influcence on the inferred lithium abundances and may, therefore, be preferable to use as reference for, e.g., model comparisons.

When comparing our inferred $\mathrm{Li}$ abundance trends with both effective temperature and stellar luminosity to predictions from stellar-structure models including atomic diffusion and ad hoc turbulence below the convection zone (cf. Richard et al. 2005), we find strong support for the need of some turbulence, with strict limits to its efficiency (cf. figure 10 in Lind et al. 2009b). In absence of macroscopic turbulence, the turnoff and subgiant stars would deplete too much lithium compared to more unevolved stars, giving rise to large $(\sim 0.6 \mathrm{dex})$ variations in surface-lithium abundance with evolutionary phase, which are not reproduced by observations. On the other hand, too efficient mixing erases any difference between the groups and cannot explain that subgiants appear more lithiumrich than less evolved stars.

\section{References}

Alonso, A., Arribas, S., \& Martínez-Roger, C. 1996, A\&A, 313, 873

Alonso, A., Arribas, S., \& Martínez-Roger, C. 1999, A\&As, 140, 261

Boesgaard, A. M. \& Steigman G. 1985, ARA\& A, 23, 319

Bennett, C. L., et al. ApJS, 148, 1

Bonifacio, P., et al. 2002, A\&A, 390, 91

Carretta, E., Gratton, R. G., Lucatello, S., Bragaglia, A., \& Bonifacio, P. 2005, A $₫ A, 433,597$

Castilho, B. V., Pasquini, L., Allen, D. M., Barbuy, B., \& Molaro, P. 2000, A\&A, 361, 92

Charbonnel, C. \& Primas, F. 2005, A\&A A, 442, 961

Charbonnel, C. \& Zahn, J.-P. 2007, A\&A, 467, L15

Cyburt, R. H., Fields, B. D., \& Olive, K. A. 2008, JCAPP, 11, 12

Dunkley, J., et al. 2009, ApJS, 180, 306

González Hernández, J. I., Bonifacio, P., Caffau, E., Steffen, M., Ludwig, H.-G., Behara, N. T., Sbordone, L., Cayrel, R., \& Zaggia, S. 2009, A\&A, 505, 13

Gustafsson, B., Edvardsson, B., Eriksson, K., Jørgensen, U. G., Nordlund, A, \& Plez, B. 2008, $A \& A, 486,951$

Korn, A. J., Grundahl, F., Richard, O., Mashonkina, L., Barklem, P. S., Collet, R., Gustafsson, B., \& Piskunov, N. 2007, ApJ, 671, 402

Lind, K., Asplund, M., \& Barklem, P. S. 2009a, A\& A, 503, 541

Lind, K., Primas, F., Charbonnel, C., Grundahl, F., \& Asplund, M. 2009b, A\&A, 503, 545

Mashonkina, L. I., Shimanskĭi, V. V., \& Sakhibullin, N. A. 2000, AstRep, 44, 790

Michaud, G., Fontaine, G., \& Beaudet, G. 1984, ApJ, 282, 206

Olive, K. A., Steigman, G., \& Walker, T. P. 2000, Phys. Rev., 333, 389

Önehag, A., Gustafsson, B., Eriksson, K., \& Edvardsson, B. 2009, A\&\&A, 498, 527

Pasquini, L. \& Molaro, P. 1996, A\&A, 307, 761

Prantzos, N. \& Charbonnel, C. 2006, $A \& A$ A, 458, 135

Ramírez, I. \& Meléndez, J. 2005, ApJ, 626, 465

Richard, O., Michaud, G., \& Richer, J. 2005, ApJ, 619, 538

Ryan, S. G., Kajino, T., Beers, T. C., Suzuki, T. K., Romano, D., Matteucci, F., \& Rosolankova, K. 2001, ApJ, 549, 55

Spergel, D. N., Verde, L., Peiris, H. V. 2003, ApJS 148, 175

Spite, M. \& Spite, F. 1982, Nature, 297, 483

Thévenin, F., Charbonnel, C., de Freitas Pacheco, J. A., Idiart, T. P., Jasniewicz, G., de Laverny, P., \& Plez, B. 2001, A\& $A, 373,905$ 